INPLASY

PROTOCOL

To cite: Liu et al. The Using of Methylene Blue Dye combined with Other Tracer in Sentinel Lymph Node Biopsy of Early Breast Cancer: A Metaanalysis. Inplasy protocol 202150107. doi:

10.37766/inplasy2021.5.0107

Received: 30 May 2021

Published: 30 May 2021

Corresponding author: Jingming Ye

md_ye@sina.com

Author Affiliation:

Breast Disease Center, Peking University First Hospital, Beijing 100034, China

Support:

YXJL-2016-0040-0065.

Review Stage at time of this submission: Data analysis.

Conflicts of interest: None declared.

\section{The Using of Methylene Blue Dye combined with Other Tracer in Sentinel Lymph Node Biopsy of Early Breast Cancer: A Meta-analysis}

Liu, HJ1; Sun, MS2; Liu, LY3; Yu, ZH4; Liu, Q5; Cheng, YJ6; Xu, L7; Liu, YH8; Ye, JM9 .

Review question / Objective: P: Sentinel lymph node biopsy (SLNB) of early breast cancer; I: methylene blue dye (MBD) combined with $99 \mathrm{~m}$ Technetium-labeled Sulphur Colloid (Tc99m) or Indocyanine Green (ICG) in SLNB; C: Only MBD in SLNB; O:SLNB's identification rate (IR), sensitivity (SEN), accuracy (AR) and reducing its false negative rate (FNR).

Eligibility criteria: (1) All cases in the included studies were diagnosed with breast cancer by cytology or histopathology; (2) At least one group in the included studies used MBD+ICG or MBD+Tc99m as dual-tracer method; (3) All cases are cNO, and there is no distant metastasis, no surgical contraindications; (4) Some or all of the following indicators can be extracted or calculated in this study: IR, SEN, AR and FNR. To evaluate the performance, the number of true positives and false negatives results can be extracted; (5) Study publication language is English or Chinese.

INPLASY registration number: This protocol was registered with the International Platform of Registered Systematic Review and Meta-Analysis Protocols (INPLASY) on 30 May 2021 and was last updated on 30 May 2021 (registration number INPLASY202150107).

\section{INTRODUCTION}

Review question / Objective: P: Sentinel lymph node biopsy (SLNB) of early breast cancer; I: methylene blue dye (MBD) combined with $99 \mathrm{~m}$ Technetium-labeled Sulphur Colloid (Tc99m) or Indocyanine
Green (ICG) in SLNB; C: Only MBD in SLNB; O:SLNB's identification rate (IR), sensitivity (SEN), accuracy (AR) and reducing its false negative rate (FNR).

Condition being studied: Sentinel lymph node biopsy (SLNB) has become the 
standard staging scheme for patients with cNO. Therefore, further improving SLNB's identification rate (IR), sensitivity (SEN), accuracy (AR) and reducing its false negative rate (FNR) have always been the concern of surgeons, and the selection of tracer is the key to the success of SLNB. The commonly tracer methods of SLNB include dye method, radioisotope method, dye combined nuclide method and fluorescent tracer method. A series of studies have verified the feasibility of using blue dye, radioisotope or fluorescent tracer alone in SLNB. The dual tracer method of blue dye combined with radioisotope or blue dye combined with fluorescent tracer is also widely used in clinical practice, and the combination of blue dye and radioisotope has been recommended by many guidelines. However, it should be noted that the use of radioisotope must be in cooperation with professional nuclear medicine, and there are problems such as prolonged hospital stay, difficult management of drugs and devices, and radiation to patients and staff. Similarly, indocyanine green started as an alternative to different sulfur blue of the blue dye is applied in clinical practice, and then it's excitation of fluorescence effect as a new kind of tracer method is applied to SLNB, however, the use of indocyanine green also need auxiliary of the infrared imaging instrument, and as the specification of tracer still lacks the evidence to a higher level, need to study in a larger patient population. In addition, many countries and regions, including China, and a large number of primary hospitals are limited in the choice of SLNB tracer by the availability of drugs, the capabilities of medical institutions, and health economics, but international guidelines and consensus are gradually increase the recommendation of the dual-tracer method to improve the IR, SEN and AR and reduce the FNR of SLNB. Under such contradictions, methylene blue dye (MBD), which is cheaper and more easily available than patent blue or isosulphan blue, combined with $99 \mathrm{~m}$ Technetium-labeled Sulphur Colloid (Tc99m) or Indocyanine Green (ICG) may be a selective and employed dual-tracer method for hospitals at different levels in these countries and regions. Therefore, this study aims to use meta-analysis to conduct a summary analysis of related studies to evaluate the IR, SEN, AR and FNR of MBD combined with Tc99m or ICG dual-tracer method in SLNB of breast cancer patients. Looking forward to provide a higher level of evidence-based medical evidence for the application of MBD combined with other tracers in the dual-tracer method in breast cancer SLNB.

\section{METHODS}

Search strategy: We conducted a comprehensive electronic literature search on PubMed, EMBASE, Web of Science, CNKI and WANFANG DATA databases from inception to September, 2020. The following Mesh terms are used: "Breast Neoplasms", "Methylene Blue" and "Sentinel Lymph Node Biopsy" and entry terms of Mesh. The Chinese database is searched with the same Chinese keywords as the English database.

Participant or population: Patients with early breast cancer (cNO).

Intervention: Methylene blue dye (MBD) combined with $99 \mathrm{~m}$ Technetium-labeled Sulphur Colloid (Tc99m) or Indocyanine Green (ICG) in SLNB in early breast cancer.

Comparator: Only MBD in SLNB in early breast cancer.

Study designs to be included: Randomised controlled trial, cohort study, and casecontrol study.Letters, editorials, case reports and reviews et. al are excluded.

Eligibility criteria: (1) All cases in the included studies were diagnosed with breast cancer by cytology or histopathology; (2) At least one group in the included studies used $M B D+I C G$ or MBD+Tc99m as dual-tracer method; (3) All cases are $\mathrm{cNO}$, and there is no distant metastasis, no surgical contraindications; (4) Some or all of the following indicators can be extracted or calculated in this study: IR, SEN, AR and FNR. To evaluate the performance, the number of true 
positives and false negatives results can be extracted; (5) Study publication language is English or Chinese.

Information sources: A systematic search of PubMed, EMBASE, Web of Science, CNKI and WANFANG DATA databases.

Main outcome(s): SLNB's identification rate (IR), sensitivity (SEN), accuracy (AR) and false negative rate (FNR).

Data management: The data was extracted by two independent reviewers (HJ. Liu and MS. Sun), and the accuracy of the data was verified by two other reviewers. Any disagreements generated are resolved through discussion. The summary information about the study characteristics includes the first author, publication year, study origin, the age of the patients, the TNM stage and the number of patients enrolled and the tracer method for SLNB.

Quality assessment / Risk of bias analysis: This Meta-analysis is suitable to use QUADAS 2 for study quality evaluation. QUADAS 2 is a standardized tool for evaluating the quality of diagnostic accuracy studies (8). This study has been revised when evaluating the quality of study. QUADAS 2 contains four fields for assessing risk and bias: Patient Selection, Index Test, Reference Standard and Flow and Timing. If the answer to all signaling questions for a domain are "yes", then risk of bias can be judged low. If any signaling question is answered "no," potential for bias exists. Review authors must then use the guidelines developed in phase 2 to judge risk of bias. The "unclear" category should be used only when insufficient data are reported to permit a judgment. then risk of biasing to the corresponding domain can be considered very low. Use similar criteria to determine applicability. All studies were independently analyzed by two independent reviewers (HJ. Liu and MS. Sun). With regard to study quality, no high risk of bias was detected in any of the included study.

Strategy of data synthesis: The metaanalysis in this study was conducted using the meta package of $R$ version 3.6.3 for Windows (http://www.R-project.org/) with calling to the JAGS software version 4.3.0 for NMA. All statistical tests were twosided, and $P$ values of less than 0.05 were deemed significant. The heterogeneity of the studies was evaluated using the inconsistency statistic (12). For outcome measures without significant heterogeneity among studies $(P>0.10)$, the fixed-effect model was used to calculate the proportion; otherwise, the random-effect model was employed. Funnel plots and Begg's tests were applied to investigate the publication bias.

Subgroup analysis: We want to simultaneously assess and compare the detection effects among the tracer methods of MBD, MBD+Tc99m and MBD+ICG. However, Studies on direct comparison between MBD+Tc99m and MBD+ICG are scarce. Therefore, we conducted a Network Meta-Aanlysis, and the random effects model was used to analyze the differences of the three tracer methods in IR, SEN, AR and FNR.

Sensitivity analysis: To overcome the effects of selection bias, some studies have managed to balance the baseline characteristics of surgical and medical cohorts with some methods, including paired design and propensity score matching. In our study, we will combine the matched and unmatched cohorts separately to verify our conclusions.

\section{Language: English.}

Country(ies) involved: China.

Keywords: Breast Cancer, Indocyanine Green (ICG), Methylene Blue, Sentinel Lymph Node Biopsy (SLNB), $99 \mathrm{~m}$ Technetium-labeled Sulphur Colloid (Tc99m).

Contributions of each author:

Author 1 - Hongjin Liu.

Email: liuhj_pkufh@163.com

Author 2 - Mingshuai Sun.

Author 3 - Liyuan Liu.

Author 4 - Zhengheng Yu. 
Author 5 - Qian Liu.

Author 6 - Yuanjia Cheng.

Author 7 - Ling Xu.

Author 8 - Yinhua Liu.

Author 9 - Jingming Ye.

Email: md_ye@sina.com 